\title{
Modelo Logístico Bayesiano no estudo do crescimento de tomates
}

\author{
Logistic Bayesian model in the study of the growth of tomatoes \\ Modelo Logístico Bayesiano en el studio del crecimiento del tomates
}

Recebido: 22/02/2021 | Revisado: 28/02/2021 | Aceito: 05/03/2021 | Publicado: 14/03/2021

Patrícia Neves Mendes

ORCID: https://orcid.org/0000-0002-0434-3241 Universidade Federal de Alfenas, Brasil

E-mail: patrícia.neves.mendes@gmail.com

Joel Augusto Muniz

ORCID: https://orcid.org/0000-0002-1069-4136 Universidade Federal de Lavras, Brasi E-mail: joamuniz@dex.ufla.br

Taciana Villela Savian

ORCID: https://orcid.org/0000-0002-3309-6075 Universidade de São Paulo, Brasil E-mail: tvsavian@usp.br

Thelma Sáfadi

ORCID: https://orcid.org/0000-0002-4918-300X Universidade Federal de Lavras, Brasil E-mail: safadi@dex.ufla.br

Gabriel da Costa Cantos Jerônimo

ORCID: https://orcid.org/0000-0001-8204-7694 Universidade Federal de Alfenas, Brasil E-mail: gabriel.ccjeronimo@gmail.com

\begin{abstract}
Resumo
Conhecer o crescimento do tomateiro e de seus frutos, medido através do acúmulo de biomassa ao longo do tempo, são fundamentais para o manejo adequado e a deteçãa de problemas no desenvolvimento das culturas. Este crescimento pode ser estudado por meio de vários modelos de regressão não linear que podem ser usados para facilitar a interpretação dos processos envolvidos no sistema de produção vegetal. Entre os modelos empíricos usados frequentemente para estimar o crescimento de plantas, e seus componentes, encontra-se a função logística. Um dos métodos utilizados para estimar os parâmetros das curvas de crescimento é o método bayesiano. Este estudo teve como objetivo aplicar a metodologia bayesiana na descrição dos dados - reais e simulados - de crescimento do diâmetro de tomates, utilizando o modelo não linear logístico. Os algoritmos para o amostrador de Gibbs e o Metropolis-Hastings foram implementados utilizando-se a linguagem R. A condição de convergência das cadeias foi verificada por meio do critério de Raftery \& Lewis, que está disponível no pacote BOA ("Bayesian Output Analysis") do software R. A metodologia bayesiana mostrou-se eficiente na estimação dos parâmetros da curva de crescimento, e as estimativas mostraram-se coerentes com os valores relatados na literatura.
\end{abstract}

Palavras-chave: Modelo não linear; Curva de crescimento; Metodologia Bayesiana.

\begin{abstract}
Knowing the growth of tomato and its fruits, as measured by biomass accumulation over time is essential for the proper handling and detection of problems in the development of crops. This growth can be studied using various models of non-linear regression that can be used to facilitate interpretation of the processes involved in plant production system. Among the empirical models often used to estimate the growth of plants and their components is the function Logistic. One method used to estimate the parameters of the growth rate is the Bayesian method. The study objective to apply the Bayesian approach in describing the data - real and simulated - the diameter growth of tomatoes, using the model Logistic. The algorithms for the Gibbs Sampler and Metropolis - Hastings were implemented using the $\mathrm{R}$ language. The condition of convergence of the chains was checked using the criteria suggested by Nogueira, Sáfadi and Ferreira (2004) available on the R software package BOA. The Bayesian approach was efficient, since it was evaluated and verified by the simulation process, with very close estimates of the parametric value, and estimates were shown to be consistent with the values reported in literature.
\end{abstract}

Keywords: Nonlinear model; Growth curve; Bayesian methodology.

\section{Resumen}

Conocer el crecimiento del tomate y sus frutos, medido por la acumulación de biomasa a lo largo del tiempo, es fundamental para el correcto manejo y detección de problemas en el desarrollo de los cultivos. Este crecimiento puede 
estudiarse mediante varios modelos de regresión no lineal que pueden utilizarse para facilitar la interpretación de los procesos involucrados en el sistema de producción vegetal. Entre los modelos empíricos que se utilizan a menudo para estimar el crecimiento de las plantas y sus componentes se encuentra la función logística. Uno de los métodos utilizados para estimar los parámetros de las curvas de crecimiento es el método bayesiano. Este estudio tuvo como objetivo aplicar la metodología bayesiana en la descripción de datos - reales y simulados - del crecimiento del diámetro del tomate, utilizando el modelo logístico no lineal. Los algoritmos muestreador de Gibbs y MetropolisHastings se implementaron utilizando el idioma R. La condición de convergencia de las cadenas se verificó utilizando el criterio de Raftery \& Lewis, que está disponible en el paquete BOA ("Bayesian Output Analysis") del software R. La metodología bayesiana demostró ser eficiente en la estimación de los parámetros de la curva de crecimiento, y las estimaciones fueron consistentes con los valores reportados en la literatura.

Palabras clave: Modelo no lineal; Curva de crecimiento; Metodología bayesiana.

\section{Introdução}

Originário da América Central e do Sul, o tomate era amplamente cultivado e consumido pelos povos précolombianos, e, atualmente, no mundo todo. Ele é o produto do desenvolvimento do ovário e do óvulo da flor formando o pericarpo e as sementes do fruto, respectivamente, após a fecundação. Apesar de ser classificado como um fruto, o tomate é estudado dentro do grupo das hortaliças, haja vista fazer parte da dieta brasileira, em conjunto com outras espécies, como parte integrante das saladas. É uma das hortaliças mais consumidas, associada, principalmente às principais refeições diárias ou nos lanches e fast -food (Conab, 2019).

O tomate é produzido em praticamente todas as regiões geográficas do Brasil e em épocas distintas sob diferentes sistemas de cultivo e diferentes níveis de manejo cultural, destacando-se como uma das hortaliças mais cultivadas. Estima-se que o tomate seja a hortaliça que ocupa a segunda posição mundial em área cultivada, e a primeira em volume industrializado. Em 2016, a safra mundial de tomate de mesa totalizou 177 milhões de toneladas em área cultivada de aproximadamente 4,8 milhões de hectares. O Brasil produziu 4,17 milhões de toneladas, em uma área de 63.980 hectares, alcançando uma produtividade média de 65,14 toneladas por hectare (FAO, 2018). Segundo o mesmo autor, o Brasil ocupa a $9^{a}$ posição mundial na produção de tomates $(2,5 \%)$, liderado pela China, Índia e Estados Unidos, que ocupam as primeiras posições, respondendo por aproximadamente por $31 \%, 11 \%$ e $8 \%$, respectivamente (Dossa \& Fuchs, 2017).

A mudança de cor do tomate é considerada como índice de colheita, porém desde que o fruto tenha completado seu desenvolvimento fisiológico este poderá ser colhido, mesmo apresentando a coloração verde clara. O tomate é um fruto climatérico e pode desenvolver cor, aroma e sabor característicos após a colheita. O ponto de colheita determina maior ou menor resistência do fruto ao manuseio, capacidade de completar a maturação, aparência e qualidade (Ferreira et al., 2004).

Desta forma, conhecer o crescimento do tomateiro e de seus frutos, medido pelo acúmulo de biomassa ao longo do tempo, é fundamental para o manejo adequado e a detecção de problemas no desenvolvimento das culturas. Assim, o desenvolvimento e o aperfeiçoamento de metodologias que permitam estudar de forma prática e eficiente este crescimento constituem uma importante fonte de pesquisa.

Uma destas metodologias pode ser representada pela análise de curvas de crescimento, a qual engloba principalmente o ajuste de modelos de regressão não linear, que podem ser usados, de acordo com suas habilidades, para facilitar a interpretação dos processos envolvidos no desenvolvimento das espécies. Tal metodologia pode ser aplicada à diversas áreas do conhecimento, como Santiago et al. (2020) que ajustaram diferentes modelos de regressão não linear a dados de mortalidade e casos de COVID-19 no Brasil, Itália e no mundo.

Entre os modelos de crescimento, destaca-se o logístico, o qual fornece informações úteis fundamentadas particularmente nas estimativas de seus parâmetros, e apresenta interpretação biológica, tais como taxas de crescimento e acúmulo máximo de biomassa (Prado et al., 2013).

Entre os diversos métodos utilizados para estimar os parâmetros das curvas de crescimento, o bayesiano mostra-se 
como um dos mais eficientes. Este consiste de informações referentes aos dados amostrais por meio da função de verossimilhança, do conhecimento prévio do pesquisador com respeito aos parâmetros do modelo traduzido por meio de distribuição a priori para os parâmetros e da obtenção da distribuição a posteriori dos parâmetros.

Diante do exposto e tendo em vista os poucos relatos de trabalhos sobre curvas de crescimento que tenham abordado, conjuntamente, os modelos não lineares e a análise bayesiana na área de Fitotecnia, este trabalho tem como objetivo aplicar o método bayesiano para o estudo desses modelos, o qual poderá ser estendido às mais variadas culturas.

\section{Materiais e Métodos}

Os dados experimentais são oriundos de um experimento, conduzido por Moura (1999), instalado segundo um delineamento casualizado em blocos, com quatro repetições. No experimento, dentre outros procedimentos, foram coletados, em 11 tempos de amostragem, dois frutos de tomate da variedade "Santa Clara" e imediatamente acondicionados em sacos de polietileno de baixa densidade e transportados para o laboratório. No laboratório foram feitas várias avaliações nos frutos, das quais, sem perda de generalidade, foi escolhida a variável diâmetro longitudinal dos frutos, para ser estudada neste trabalho.

Aos dados de diâmetro longitudinal do fruto do tomateiro foi ajustado o modelo logístico em função das épocas de colheita em dias após a antese (d.a.a). O modelo é representado por:

$$
\boldsymbol{Y}_{i}=\frac{A}{\left(1+B e^{\left.-K t_{i}\right)}\right.}+\varepsilon_{i}
$$

em que: Yi representa o diâmetro longitudinal do fruto em milímetros com $\mathrm{i}=1, \ldots, \mathrm{n}$ e $\mathrm{n}$ o número de medições no fruto; A é o parâmetro assintótico que corresponde ao diâmetro do fruto à maturação; B corresponde ao parâmetro de locação, sem interpretação biológica; K determina a taxa de expansão do crescimento; ti refere-se ao i-ésimo tempo de amostragem, dado em dias após a antese; عi é o resíduo no tempo i, considerado independente e identicamente distribuído de uma Normal com média zero e variância constante $\sigma^{2}$ e n é o número de observações.

Para a obtenção da distribuição a posteriori conjunta dos parâmetros do modelo logístico, assumiram-se prioris uniformes para os parâmetros A, B e K. Para a precisão $\tau$, dada pelo inverso da variância $\sigma^{2}$, assumiu-se como priori uma distribuição Gama com hiperparâmetros $\alpha$ e $\beta$ cujos valores foram, respectivamente, 50.000 e 100.000 . As distribuições $a$ priori foram especificadas com base em resultados de estudos anteriores que utilizaram conjuntos de dados semelhantes e o mesmo modelo, pois este processo de atualização de informação via análises prévias constitui uma importante característica da análise Bayesiana. A função de verossimilhança dos parâmetros é dada por:

$$
L(Y, A, B, K, \tau, \alpha, \beta) \propto \tau^{\frac{n}{2}} \exp \left\{\frac{-\tau}{2} \sum_{i=1}^{n}\left[y_{i}-A\left[1+\exp \left(-K t_{i}\right)^{-1}\right]\right]^{2}\right\}
$$

em que $\mathrm{Y}=\{\mathrm{y} 1, \ldots, \mathrm{yn}\}$. Por meio do teorema de Bayes, a distribuição a posteriori conjunta é dada por:

$$
p(A, B, K, \tau, Y, \alpha, \beta) \propto \tau^{\frac{n}{2}} \exp \left\{\frac{-\tau}{2} \sum_{i=1}^{n}\left[y_{i}-A\left[1+\exp \left(-K t_{i}\right)^{-1}\right]\right]^{2}\right\} \tau^{\alpha-1} \exp (-\beta \tau)
$$

Com o intuito de analisar o procedimento bayesiano na estimação dos parâmetros do modelo considerou-se no presente trabalho um estudo com dados simulados a partir do modelo logístico. No estudo foram obtidos 77 valores que 
correspondem a mensurações diárias, iniciando aos 7 dias após a antese, do diâmetro longitudinal de tomates. Os valores paramétricos escolhidos com base em estudos com a variedade "Santa Clara" e assumidos como verdadeiros para o modelo Logístico foram: A (valor de estabilização do crescimento do diâmetro dos tomates) igual a $50 \mathrm{~mm}$; B (constante, sem interpretação biológica) igual a 3; K (taxa de crescimento da variável dependente) igual a 0,05 mm/dia e $\varepsilon$ i seguindo uma distribuição normal com média zero e variância 2 , ou seja, $\varepsilon i \sim \mathrm{N}(0,2)$.

Para a análise dos dados, experimentais e simulados, utilizou-se o software R (R development core team, 2019), no qual foi implementado o algoritmo para o Amostrador de Gibbs e o Metropolis-Hastings. Na análise dos dados simulados foram consideradas 40.000 iterações das quais $10 \%$ foram eliminadas (burn-in), ou seja, 4.000 delas foram descartadas a fim de evitar os efeitos dos valores iniciais dos parâmetros adotados. Além disso, considerou-se um espaçamento entre os pontos amostrados (thin) de tamanho 10. Desta forma, obteve-se uma cadeia final de 3.600 observações para cada parâmetro do modelo. A verificação final da convergência das cadeias foi feita utilizando o pacote BOA do software R.

$\mathrm{Na}$ análise dos dados reais foram consideradas 70.000 iterações, das quais foram descartadas as 1.000 primeiras iterações e para assegurar a independência da amostra, utilizou-se também um salto de tamanho 10 entre as observações da cadeia. Sendo assim, a cadeia final ficou composta por 6.900 observações. Para a verificação final da convergência das cadeias foram utilizados os mesmos critérios dos dados simulados.

Foram obtidas estimativas pontuais (média, moda) e intervalares por meio do intervalo de credibilidade de máxima densidade de probabilidade (highest probability density-HPD) para os parâmetros dos modelos.

\section{Resultados e Discussão}

A partir da distribuição a posteriori conjunta dos parâmetros foram obtidas as distribuições condicionais completas para cada parâmetro, a fim de obter aproximações das distribuições marginais dos parâmetros. Para o parâmetro A, que representa o diâmetro assintótico do fruto, a distribuição condicional completa a posteriori é dada por:

$$
p(A, B, K, \tau, Y, \alpha, \beta) \propto \exp \left\{\frac{-\tau}{2}\left(\sum_{i=1}^{n}\left[\left(1+B \exp \left(-K t_{i}\right)^{-1}\right)\right]^{2}\right)\left[-2 A \frac{\sum_{i=1}^{n} y_{i}\left(1+B \exp \left(-K \mathrm{t}_{i}\right)\right)^{-1}}{\sum_{i=1}^{n}\left(\left[1+B \operatorname{sxp}\left(-K \mathrm{t}_{i}\right)\right]^{-1}\right)^{2}}\right]+A^{2}\right\}
$$

Essa expressão representa o núcleo de uma distribuição normal com média e variância dadas, respectivamente, por:

$$
\mu=\frac{\sum_{i=1}^{n} y_{i}\left[1+B \exp \left(-K t_{i}\right)\right]^{-1}}{\sum_{i=1}^{n}\left(\left[1+B \exp \left(-K t_{i}\right)\right]^{-1}\right)^{2}}
$$

e

$$
\sigma^{2}=\frac{\sigma^{2} e}{\sum_{i=1}^{\mathfrak{n}}\left(\left[\mathbf{1}+\operatorname{Bexp}\left(-K t_{i}\right)\right]^{-1}\right)^{2}}
$$

A distribuição condicional completa a posteriori para o parâmetro B do modelo Logístico pode ser escrita, proporcionalmente, como: 


$$
p(B, A, K, \tau, Y, \alpha, \beta) \propto \exp \left\{\frac{-\tau}{2}\left(\sum_{i=1}^{\mathfrak{n}}\left(y_{i}-A\left[1+B \exp \left(-K t_{i}\right)\right]^{-1}\right)^{2}\right)\right\}
$$

Considerando o parâmetro K do modelo Logístico, sua distribuição condicional completa a posteriori é dada por:

$$
p(K, A, B, \tau, Y, \alpha, \beta) \propto \exp \left\{\frac{-\tau}{2}\left(\sum_{i=1}^{n}\left(y_{i}-A\left[1+B \exp \left(-K t_{i}\right)\right]^{-1}\right)^{2}\right)\right\}
$$

Para os parâmetros $\mathrm{B}$ e $\mathrm{K}$ as distribuições condicionais completas a posteriori não têm forma conhecida sendo necessária, nesses casos, a utilização do algoritmo de Metropolis-Hastings para geração das cadeias. O algoritmo usa a ideia de que um valor é gerado de uma distribuição auxiliar e aceito com uma dada probabilidade.

Considerando a análise dos dados simulados, a distribuição auxiliar para o parâmetro B foi uma distribuição Normal com média 3 e variância 1; para o parâmetro K, a distribuição auxiliar foi uma Gama com os valores dos parâmetros alfa e beta iguais a 5.000 e 100.000, respectivamente. Na análise dos dados reais, considerou-se para o parâmetro B uma distribuição auxiliar Normal com média 5 e variância 1 e para o parâmetro K, considerou-se uma distribuição Gama de parâmetros 700 e 10.000 para alfa e beta, respectivamente.

A distribuição condicional completa a posteriori para o parâmetro é escrita, de forma proporcional, como:

$$
p(\tau, A, B, K, Y, \alpha, \beta) \propto \tau^{\frac{n+2 \alpha}{2}-1} \exp \left\{\left[-\tau\left(\beta+\frac{1}{2} \sum_{i=1}^{n}\left(y_{i}-A\left[1+B \exp \left(-K t_{i}\right)\right]^{-1}\right)^{2}\right)\right]\right\}
$$

Esta expressão representa o núcleo de uma distribuição Gama com os seguintes parâmetros:

$$
\alpha^{*}=\frac{n+2 \alpha}{2}
$$

$\mathrm{e}$

$$
\beta^{*}=\beta+\frac{1}{2} \sum_{i=1}^{n}\left(y_{i}-A\left[1+B \exp \left(-K t_{i}\right)\right]^{-1}\right)^{2}
$$

sendo $\alpha$ e $\beta$ os hiperparâmetros da distribuição a priori para o parâmetro $\tau$, que assumiram os valores de 25.000 e 50.000, para a análise dos dados simulados; e os valores de 500 e 1.000 quando se analisaram os dados reais.

De acordo com os resultados apresentados na Tabela 1, é possível perceber que as estimativas da média e moda a posteriori, para o parâmetro K, são iguais, indicando uma distribuição do tipo simétrica para este parâmetro.

Com relação aos outros parâmetros, a proximidade entre as estimativas da média e moda a posteriori é menor, fato que também pode ser observado nas Figuras de 1, 2 e 4. Em situações como esta, o melhor valor pontual para representar os dados é a moda a posteriori.

Ainda na Tabela 1, observa-se que os intervalos de máxima densidade a posteriori estimados são todos estatisticamente significativos. Percebe-se também que a metodologia bayesiana foi eficaz na estimação dos parâmetros do modelo logístico, estando todos os valores paramétricos utilizados no cenário da simulação dentro dos intervalos de máxima densidade a posteriori. Desta forma, pode-se considerar que a metodologia utilizada se adapta às diversas situações encontradas em estudo de crescimento de frutos. 
Tabela 1 - Valores paramétricos, estimativa da média e moda a posteriori, intervalo de máxima densidade a posteriori (HPD) e critérios de Geweke (valor p) e Gelman \& Rubin $(R)$ utilizados na detecção da convergência das cadeias amostradas para os dados simulados do modelo logístico.

\begin{tabular}{|c|c|c|c|c|c|c|c|}
\hline \multicolumn{2}{|c|}{ Valores paramétricos } & \multirow[t]{2}{*}{ Média } & \multirow[t]{2}{*}{ Moda } & \multicolumn{2}{|c|}{ Intervalo HPD } & \multirow{2}{*}{$\begin{array}{l}\text { Geweke } \\
\text { (valor-p) }\end{array}$} & \multirow{2}{*}{$\begin{array}{c}\text { Gelman \&Rubin } \\
\widehat{R}\end{array}$} \\
\hline & & & & LI & $\mathbf{L S}$ & & \\
\hline$a$ & 50 & 49,36 & 49,71 & 41,62 & 56,42 & 0,0903 & 0,9996 \\
\hline$b$ & 3,0 & 2,86 & 2,91 & 0,75 & 4,49 & 0,8776 & 0,9994 \\
\hline$k$ & 0,05 & 0,05 & 0,05 & 0,04 & 0,05 & 0,5763 & 0,9994 \\
\hline$T$ & 0,5 & 0,49 & 0,49 & 0,47 & 0,508 & 0,7691 & 0,9994 \\
\hline
\end{tabular}

Fonte: Autores.

Reis et al. (2011) encontraram intervalos de credibilidade contendo os valores reais de cada parâmetro, para quase todos os cenários estudados, em trabalhos de simulação feitos para comparação bayesiana de modelos aplicados ao equilíbrio de Hardy-Weinberg. Resultados semelhantes foram obtidos também por Pereira et al. (2009) em estudo bayesiano da predição de nitrogênio mineralizado.

Em relação aos critérios de convergência das cadeias, observa-se, ainda na Tabela 1, que todas as cadeias geradas pelo Método de Monte Carlo via cadeias de Markov (MCMC) atingiram a convergência para o cenário simulado no modelo logístico. De acordo com o critério de Geweke (1992), as cadeias relativas a todos os parâmetros convergiram, uma vez que os valores-p encontrados foram maiores que o nível de significância pré-estabelecido em 0,05. Observando-se o parâmetro A, especificamente, percebe-se que o valor-p encontrado foi de 9,03\%, sendo este resultado ainda assim superior ao mínimo estabelecido e suficiente para indicar a convergência desta cadeia.

Os valores obtidos para o fator de redução R do critério de Gelman \& Rubin (1992) também apontam para a convergência das cadeias, estando todos eles muito próximo de 1.

Nas Figuras de 1 a 4 abaixo são apresentados os traços das cadeias para os parâmetros do modelo logístico e suas respectivas densidades a posteriori.

Observa-se que as cadeias geradas para os parâmetros apresentam um comportamento que oscila em torno das médias estimadas, sem picos ao longo da cadeia. Pode-se observar ainda, a simetria da cadeia para o parâmetro K, na Figura 3, fato já indicado e relatado anteriormente, quando foi feita a análise das médias e modas a posteriori. Para os outros parâmetros, ilustrados nas Figuras 1, 2 e 4 observa-se que as cadeias variaram um pouco em torno de seus respectivos valores paramétricos e também nota-se a assimetria apresentada por estes parâmetros. 
Figura 1 - Densidade a posteriori e traço da cadeia gerada pelo método MCMC para o parâmetro $A$ do modelo logístico, referentes à simulação.
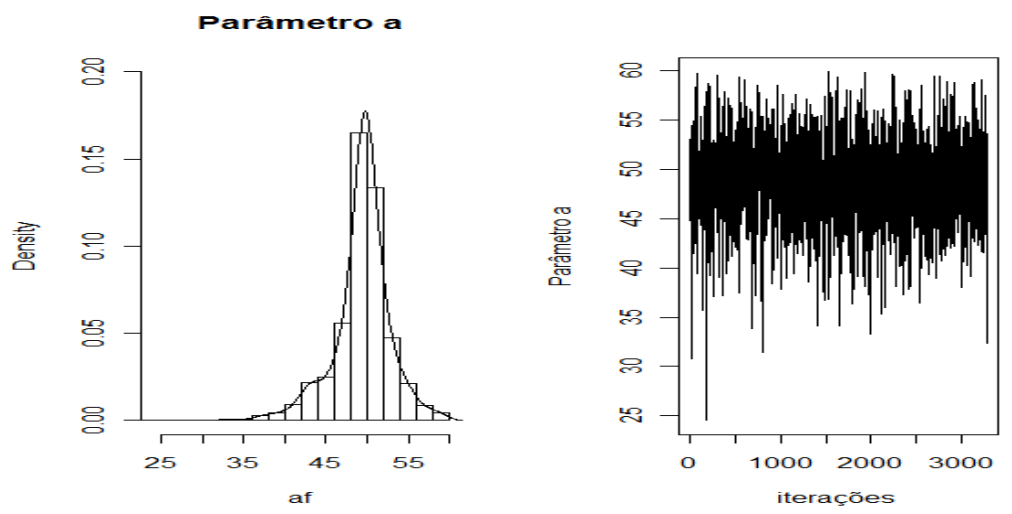

Fonte: Autores.

Figura 2 - Densidade a posteriori e traço da cadeia gerada pelo método MCMC para o parâmetro $B$ do modelo logístico, referentes à simulação.
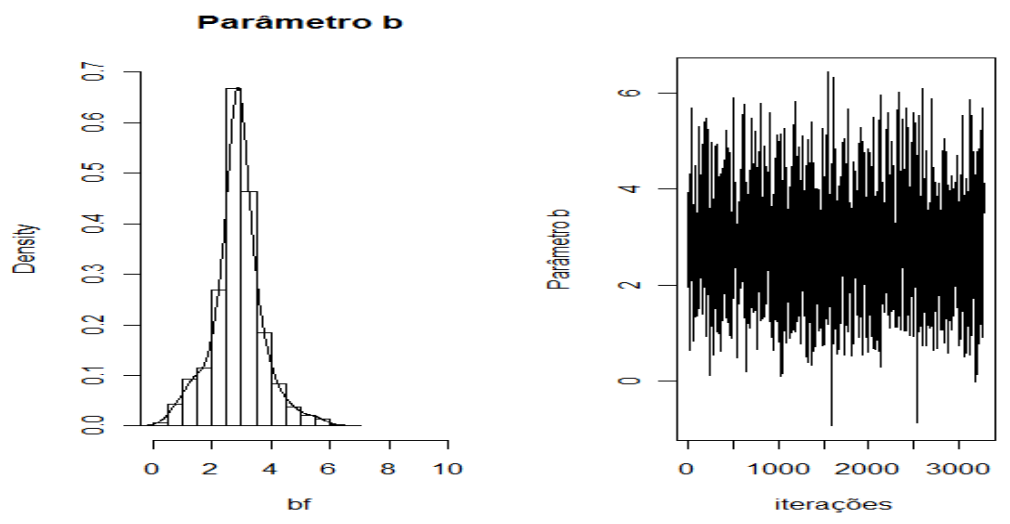

Fonte: Autores.

Figura 3 - Densidade a posteriori e traço da cadeia gerada pelo método MCMC para o parâmetro $K$ do modelo logístico, referentes à simulação.
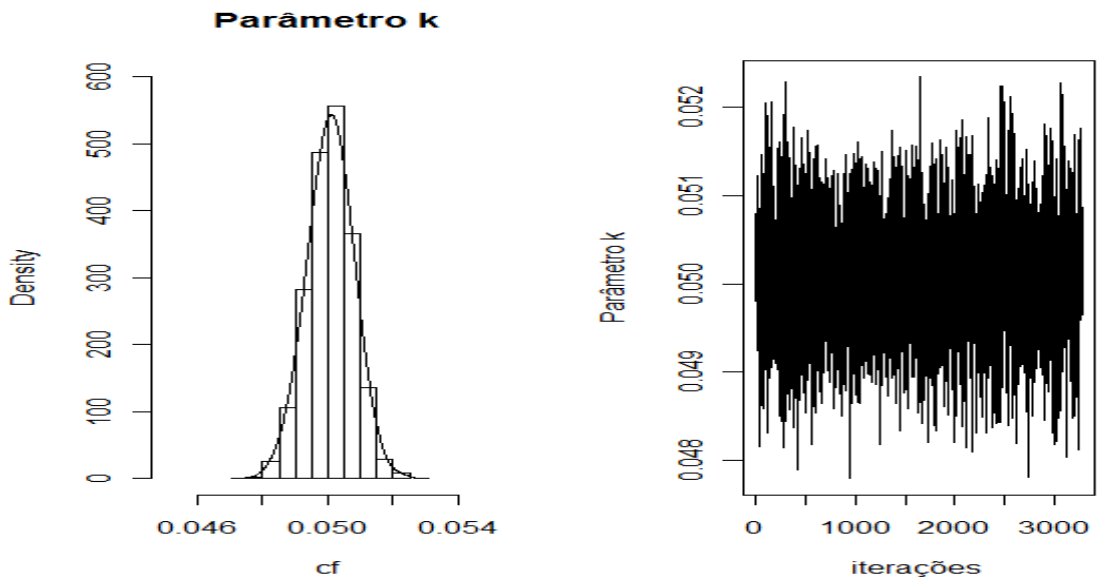

Fonte: Autores. 
Figura 4 - Densidade a posteriori e traço da cadeia gerada pelo método MCMC para o parâmetro do modelo logístico, referentes à simulação.
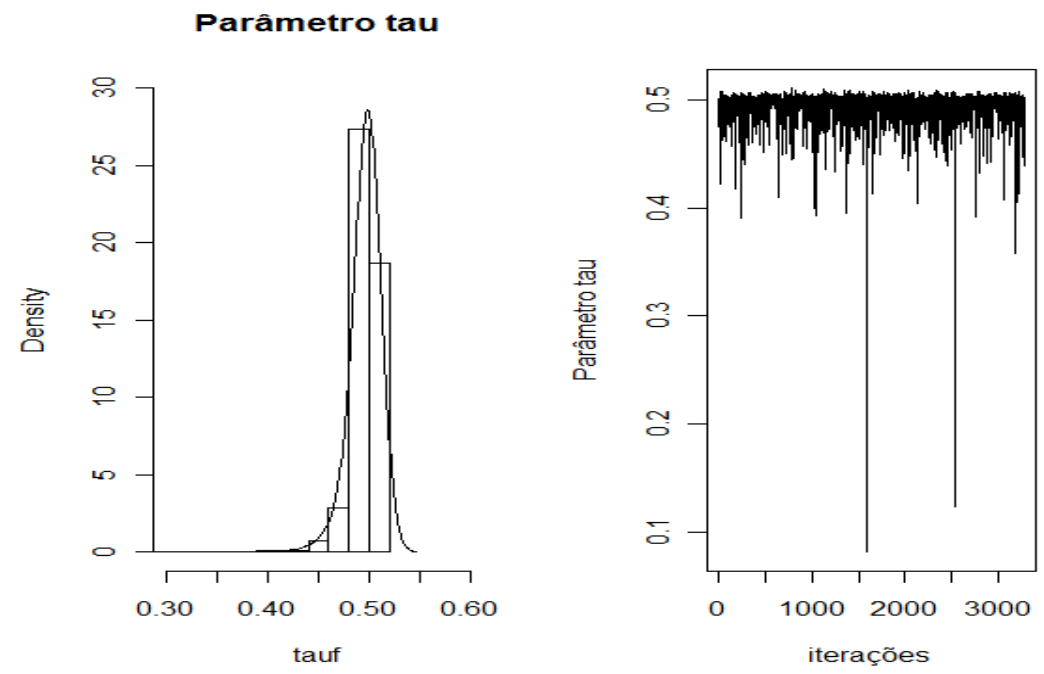

Fonte: Autores.

De acordo com os resultados apresentados na Tabela 2 para a análise dos dados reais, percebe-se que as estimativas da média e moda a posteriori mostram-se bem próximas entre si, sugerindo distribuições do tipo simétricas para os parâmetros envolvidos no modelo. Savian et al. (2009) também encontraram estimativas da média e moda a posteriori bem próximas entre si, evidenciando distribuições do tipo simétricas para todos os parâmetros, num estudo feito utilizando a metodologia bayesiana na comparação de modelos de degradabilidade ruminal.

Com base nos valores estimados para o parâmetro a pode-se dizer que o modelo logístico estimou o diâmetro longitudinal dos tomates em valores condizentes aos relatados para cultivar "Santa Clara" (em torno de $77 \mathrm{~mm}$ ). O valor médio para o parâmetro $\mathrm{K}$ foi estimado em $0,0736 \mathrm{~mm} /$ dia, ou seja, essa é a taxa de expansão do crescimento dos frutos de tomateiro. Moura et al. (2004) utilizaram a metodologia clássica para ajustar os modelos e obtiveram, para taxa de expansão (K), um valor estimado em $0,1142 \mathrm{~mm} /$ dia. Isto significa que no presente estudo, utilizando a metodologia bayesiana, os frutos demoraram mais tempo a atingir a maturidade, isto é, seu crescimento foi mais lento. No estudo de curvas de crescimento de duas cultivares de feijoeiro, Martins Filho et al. (2008) verificaram que a cultivar "carioca" apresentou maior precocidade que a cultivar "neguinho", pois os valores encontrados para o parâmetro K na primeira cultivar foram maiores que os valores do cultivo de "neguinho", no ajuste do modelo logístico aos dados de altura das plantas.

No estudo realizado por Pereira et al. (2000) sobre o crescimento da bananeira "Prata-Anã", o modelo logístico foi ajustado aos dados de altura das plantas e observou-se que as bananeiras tinham cerca de 0,16m de altura à época do plantio. Por volta do sexto mês, as plantas atingiram metade da altura máxima, e aos nove meses, se encontravam com 2,65m, tendendo à estabilidade a partir daí. A taxa de crescimento atingiu seu valor máximo por volta do sexto mês após o plantio, com o valor de $0,48 \mathrm{~m} / \mathrm{mês}$.

Pode-se observar que a metodologia utilizada foi eficiente, uma vez que todos os intervalos de máxima densidade a posteriori (HPD) foram significativos, pois nesse intervalo todos os valores têm a mesma probabilidade de ocorrência. Entre todos os intervalos estimados, nota-se que a maior dispersão foi no parâmetrơ . Pelos valores encontrados nas estimativas dos parâmetros, uma informação pontual pode ser obtida tanto pelo valor da média, quanto pelo valor da moda a posteriori, 
pois ambos os valores estão muito próximos entre si.

Tabela 2 - Estimativas da média e moda a posteriori, intervalo de máxima densidade a posteriori (HPD) e critérios de Geweke (valor p) e Gelman \& Rubin (R) utilizados na detecção da convergência das cadeias amostradas para os dados reais do modelo logístico.

\begin{tabular}{ccccccc}
\hline Parâmetros & Média & Moda & \multicolumn{2}{c}{ Intervalo HPD } & Geweke & Gelman \&Rubin \\
\cline { 4 - 5 } & & & LI & LS & (valor-p) & $\widehat{\widehat{\boldsymbol{R}}}$ \\
\hline$a$ & 77,0934 & 77,1723 & 75,1001 & 79,2287 & 0,1919 & 1,0050 \\
$b$ & 7,5739 & 7,6656 & 6,5029 & 8,8094 & 0,1509 & 1,0107 \\
$k$ & 0,0736 & 0,0741 & 0,0693 & 0,0781 & 0,7928 & 1,0046 \\
$\boldsymbol{T}$ & 0,4608 & 0,4631 & 0,4176 & 0,5042 & 0,6312 & 1,0029 \\
\hline
\end{tabular}

Fonte: Autores.

Ainda na Tabela 2 são apresentados os resultados relacionados à convergência das cadeias do modelo logístico ajustado aos dados reais. Observa-se que todas as cadeias geradas pelo método MCMC atingiram a convergência. Mais uma vez, os valores obtidos para o critério de Geweke (1992) apontam para a convergência das cadeias, encontrando valores-p maiores que 0,05 . Os valores alcançados para o fator de redução R do critério de Gelman \& Rubin (1992) também indicam que as cadeias convergiram, uma vez que este critério trabalha com resultados de R satisfatórios próximos do valor 1.

A visualização das Figuras de 5 a 8 possibilita a confirmação dos resultados relacionados à convergência das cadeias apresentados na Tabela 2. Observando-a, percebe-se que, com exceção do parâmetro $B$, todos os outros apresentam uma distribuição com tendência à simetria, fato já observado pela proximidade das estimativas. O mesmo não ocorre com o parâmetro $B$ para o qual se observou uma distribuição assimétrica à esquerda.

Figura 5 - Densidade a posteriori e traço da cadeia geradas pelo método MCMC para o parâmetro $a$ do modelo logístico, referente aos dados reais.
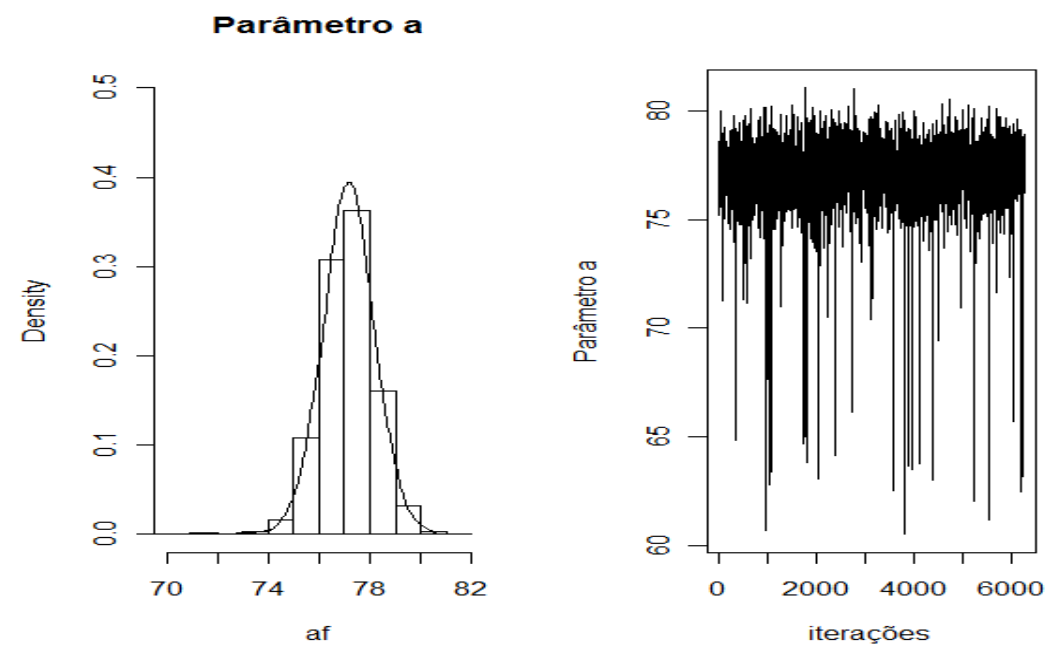

Fonte: Autores.

Figura 6 - Densidade a posteriori e traço da cadeia geradas pelo método MCMC para o parâmetro $b$ do modelo logístico, 
referente aos dados reais.
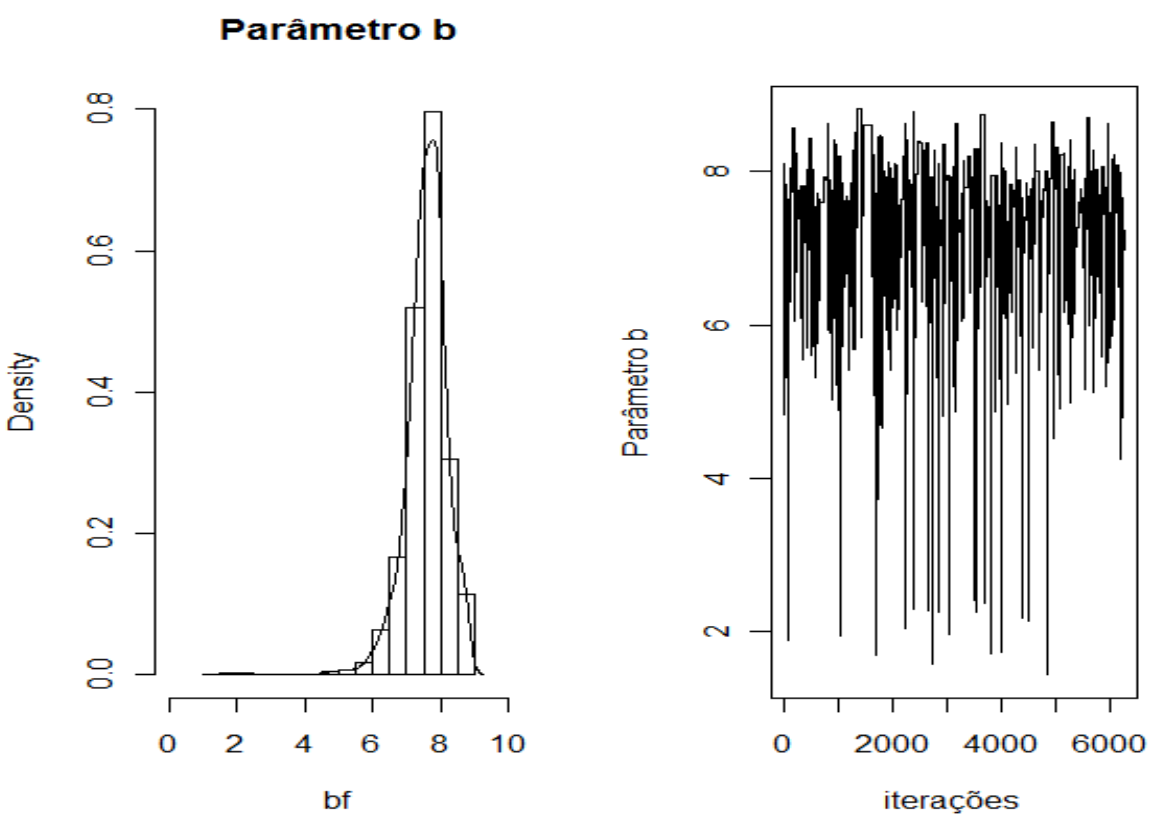

Fonte: Autores.

Figura 7 - Densidade a posteriori e traço da cadeia geradas pelo método MCMC para o parâmetro $k$ do modelo logístico, referente aos dados reais.
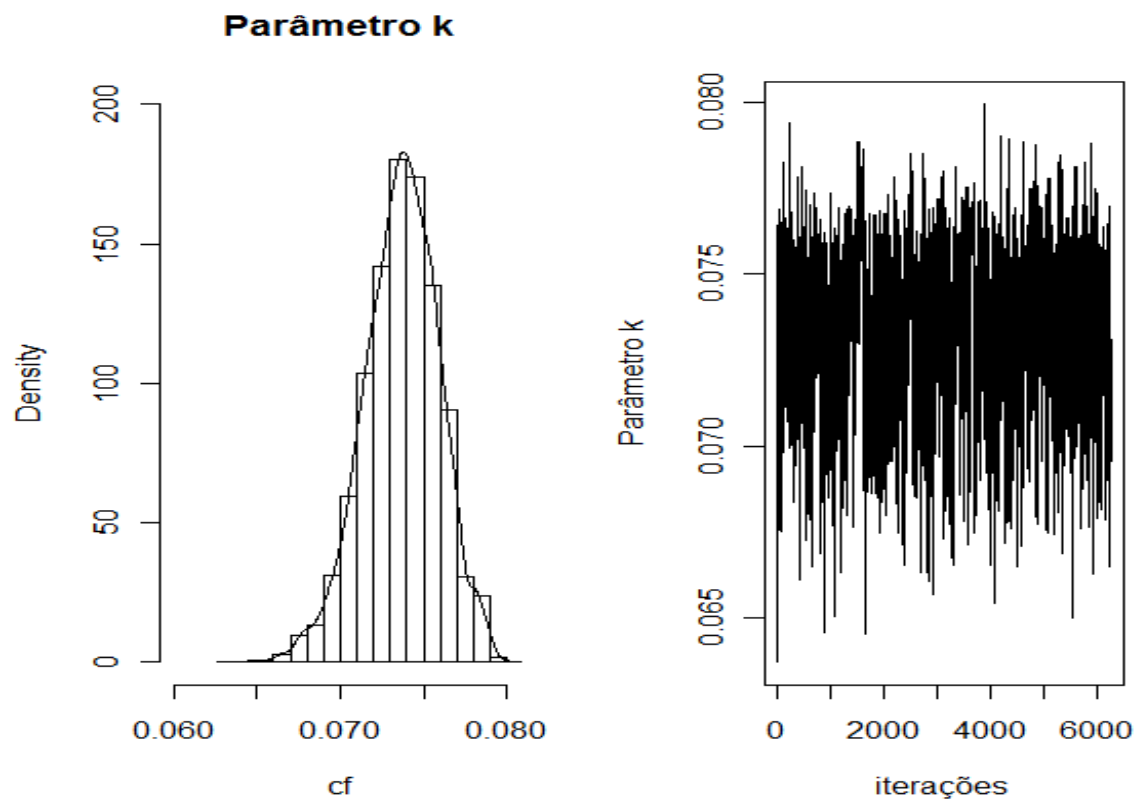

Fonte: Autores. 
Figura 8 - Densidade a posteriori e traço da cadeia gerada pelo método MCMC para o parâmetro do modelo logístico, referente aos dados reais.

\section{Parâmetro tau}
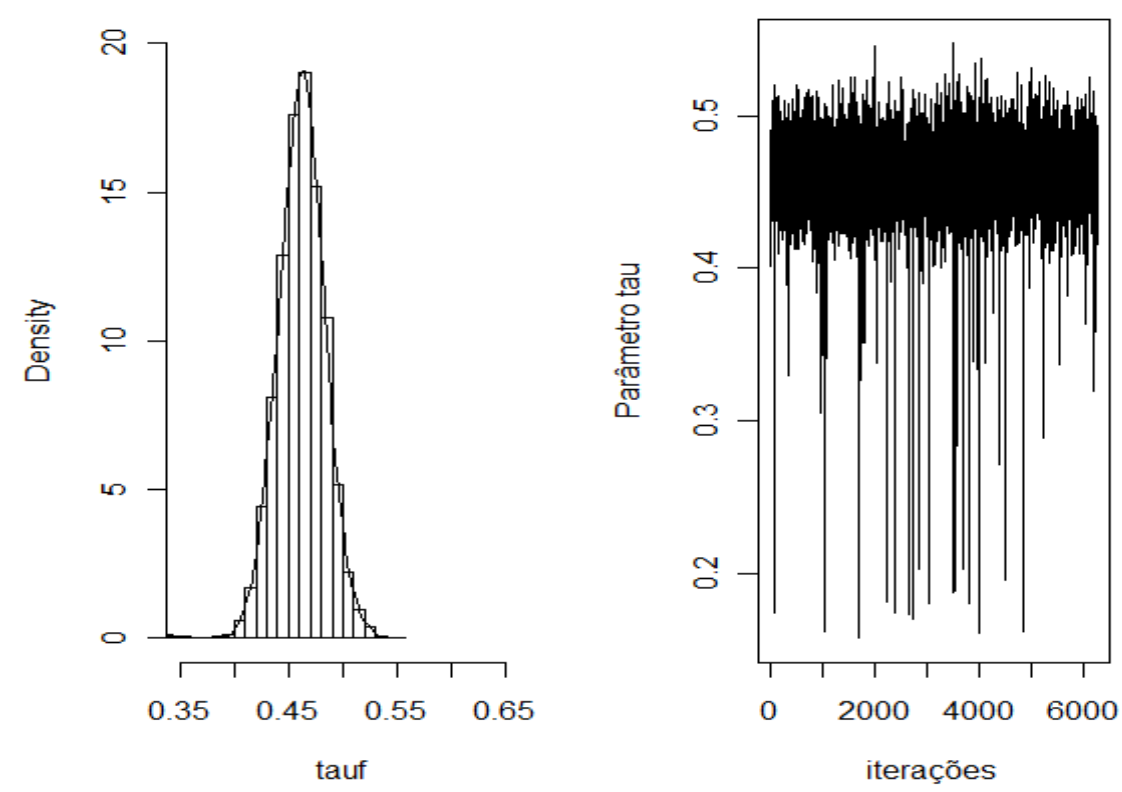

Fonte: Autores.

\section{Conclusão}

De acordo com os resultados obtidos para o ajuste do modelo aos dados do diâmetro longitudinal de frutos de tomateiro, conclui-se que o modelo Logístico apresentou valores para as estimativas dos parâmetros coerentes com a realidade dos frutos de tomateiro do tipo 'Santa Clara'.

Nota-se também que a metodologia bayesiana mostrou-se eficiente no estudo de crescimento de tomates, fato comprovado pelo estudo de simulação realizado no estudo. Desta forma, a metodologia bayesiana pode ser explorada em experimentos futuros e aplicadas as mais diversas culturas.

\section{Referências}

Companhia Nacional de Abastecimento [CONAB] (2019). Tomate: Análise dos indicadores da produção e comercialização no mercado mundial, brasileiro e catarinense. https://www.conab.gov.br/institucional/publicacoes/compendio-de-estudos-da-conab/item/download/29586_4fe6dd2c9c6d1fa5e1cbc5f82061717d

Dossa, D.; Fuchs, F. (2017). Tomate: análise técnico-econômica e os principais indicadores da produção nos mercados mundiais, brasileiro e paranaense. Boletim Técnico. http://www.ceasa.pr.gov.br/arquivos/File/BOLETIM/Boletim_Tecnico_Tomate1.pdf

Food and Agriculture Organization of the United Nations [FAO] (2018). http://www.fao.org/faostat/en/\#home

Ferreira, S. M. R., Freitas, R. J. S., \& Lazzari, E. N. (2004). Padrão de identidade e qualidade do tomate (Lycopersicon esculentum Mill.) de mesa. Ciência Rural, 34(1), 329-335. 10.1590/S0103-84782004000100054

Gelman, A., \& Rubin, D. B. (1992). Inference from iterative simulation using multiple sequences. Statistical Science.

Geweke, J. (1992). Evaluating the accuracy of sampling-based approaches to calcualting posterior moments. In: Bernardo, J. M. et al. (Ed.). Bayesian statistics.

Martins Filho, S., Silva, F. F. e., Carneiro, A. P. S., \& Muniz, J. A. (2008). Abordagem Bayesiana das curvas de crescimento de duas cultivares de feijoeiro. Ciência Rural, 38(6), 1516-1521. 10.1590/S0103-84782008000600004.

Moura, M. A.de. (1999). Crescimento pós-colheita de frutos de tomateiro cv. Santa Clara e do seu Mutante Firme. 1999. 86p. Dissertação (Mestrado em Agronomia) - Universidade Federal de Viçosa, Viçosa, MG. 
Research, Society and Development, v. 10, n. 3, e22710313198, 2021

(CC BY 4.0) | ISSN 2525-3409 | DOI: http://dx.doi.org/10.33448/rsd-v10i3.13198

Moura, M. L., Fogaça, C. M., Moura, M. A., Galvão, H. L., \& Finger, F. L. (2004). Crescimento e desenvolvimento de frutos de tomateiro "Santa Clara" e do seu mutante natural "Firme". Ciência e Agrotecnologia, 28(6), 1284-1290. 10.1590/S1413-70542004000600009

Nogueira, D. A., Safadi, T., \& Ferreira, D. F. (2004). Avaliação de critérios de convergência para o método de Monte Carlo via Cadeias de Markov. Revista Brasileira de Estatística, 65(224), 59-88.

Pereira, J. M., Muniz, J. A., Safadi, T., \& Silva, C. A. (2009). Comparação entre modelos para predição do nitrogênio mineralizado: uma abordagem bayesiana. Ciência e Agrotecnologia, 33, 1792-1797. 10.1590/S1413-70542009000700016

Pereira, M. C. T., Salomão, L. C. C., Silva, S. O. e., Sediyama, C. S., Couto, F. A. A., \& Silva Neto, S. P. da. (2000). Crescimento e produção de primeiro ciclo da bananeira "Prata-Anã" (AAB) em sete espaçamentos. Pesquisa Agropecuária Brasileira, 35(7), 1377-1387. 10.1590/S0100-204X2000000700012

Prado, T. K. L. do., Savian, T. V., \& Muniz, J. A. (2013). Ajuste dos modelos Gompertz e Logístico aos dados de crescimento de frutos de coqueiro anão verde. Ciência Rural, 43(5), 803-809. 10.1590/S0103-84782013005000044.

R development core team. R: a language and environment for statistical computing. 2019.

Raftery, A. L., \& Lewis, S. (1992). Comment: one long run with diagnostics: implementation strategies for Markov chain Monte Carlo. Statistical Science.

Reis, R. L., Muniz, J. A., Silva, F. F. e., Safadi, T., \& Aquino, L. H. (2001). Comparação bayesiana de modelos com uma aplicação para o equilíbrio de HardyWeinberg usando o coeficiente de desequilíbrio. Ciência Rural, 41(5), 834-840. 10.1590/S0103-84782011000500016

Santiago, E. J. P., Freire, A. K. S., Cunha Filho, M., Moreira, G. R., Ferreira, D. S. A., \& Cunha, A. L. X. (2020). Modelos não lineares aplicados a mortalidade e casos da COVID-19 no Brasil, Itália e mundo. Research, Society and Development. http://dx.doi.org/10.33448/rsd-v9i6.3561

Savian, T. V. et al. Análise bayesiana para modelos de degradabilidade ruminal. (2009). Ciência Rural, 39(7), 2169-2177. 10.1590/S010384782009000700033 